\title{
A Cross-Sectional Study on Appetite, Nutritional Status and Nutritional Support of Hospitalized Patients
}

\author{
Youdi Cai', Meinong Zhong1, Ya Jiang2, Xueling Zhou ${ }^{3}$, Xiaolan $\mathrm{He}^{3}$, Qian Luo ${ }^{4}$, Xiaoling Li5, \\ Shiju Huang ${ }^{*}$
}

\begin{abstract}
${ }^{1}$ Department of Urology, The Third Affiliated Hospital of Sun Yat-sen University, Guangzhou, China ${ }^{2}$ Department of Nursing, The Third Affiliated Hospital of Sun Yat-sen University, Guangzhou, China ${ }^{3}$ Department of Gastrointestinal Surgery, The Third Affiliated Hospital of Sun Yat-sen University, Guangzhou, China ${ }^{4}$ Oral and Optic Plastic Surgery Ward, The Third Affiliated Hospital of Sun Yat-sen University, Guangzhou, China ${ }^{5}$ Department of Nutrition, The Third Affiliated Hospital of Sun Yat-sen University, Guangzhou, China ${ }^{6}$ Department of Surgery, The Third Affiliated Hospital of Sun Yat-sen University, Guangzhou, China Email: 417192990@qq.com, *huangshiju2000@163.com
\end{abstract}

How to cite this paper: Cai, Y.D., Zhong, M.N., Jiang, Y., Zhou, X.L., He, X.L., Luo, Q., Li, X.L. and Huang, S.J. (2021) A CrossSectional Study on Appetite, Nutritional Status and Nutritional Support of Hospitalized Patients. Open Journal of Nursing, 11, 1002-1014.

https://doi.org/10.4236/ojn.2021.1111080

Received: October 13, 2021

Accepted: November 26, 2021

Published: November 29, 2021

Copyright $\odot 2021$ by author(s) and Scientific Research Publishing Inc. This work is licensed under the Creative Commons Attribution International License (CC BY 4.0).

http://creativecommons.org/licenses/by/4.0/

\begin{abstract}
Objective: To understand the appetite and nutritional status of hospitalized patients in a tertiary A general hospital in Guangzhou, Guangdong Province. Methods: A cross-sectional survey of appetite and nutritional status assessment on inpatients in 44 wards of the hospital was conducted. Taking all "conscious patients hospitalized for more than 48 hours" in the hospital on November 25, 2020 as the survey subjects, the patients' appetite, dietary intake, nutrition and nutritional support in the past week were investigated. Results: A total of 890 cases were investigated, among which 25 cases $(2.81 \%)$ with missing data were excluded, and thus 865 investigated cases were considered effective. The incidence of nutritional risk was $28.67 \%$, malnutrition 13.29\%, external tube feeding nutrition $3.24 \%$, parenteral nutrition $7.05 \%$, and oral nutritional supplement $10.40 \%$. The average score of appetite assessment was $(6.99 \pm 2.43)$ points. Among them, cases with appetite assessment scores $<5$ points accounted for $15.84 \%$, and 52 patient cases utilized appetite-improving drugs. Among the 137 patients with appetite scores $<5$, only 7 patients utilized appetite-improving drugs. The patients' dietary selfevaluation scores were averagely $(4.08 \pm 1.16)$ points, and the daily intake compliance rate of patients was $85.78 \%$. Appetite assessment score was correlated with dietary intake score $(r=0.548)$ and daily intake compliance rate $(r$ $=0.263)(p<0.01)$. The differences in body weight, BMI, grip strength, albumin, and hemoglobin concentration of patients with different appetite states were statistically different $(p<0.01)$. Appetite was an influencing factor of
\end{abstract}


weight change ( $\beta=-0.079, p=0.023)$. The difference between the appetite assessment scores and the daily intake compliance rates of patients with different nutritional support methods was statistically significant $(p<0.05)$; the individualized diet group had the highest appetite assessment score $(8.57 \pm$ 1.70), while the parenteral nutrition group had the lowest appetite assessment score $(4.90 \pm 2.99)$; the individualized diet group had the highest daily intake rate of $100 \%$, followed by the parenteral nutrition group with $96.72 \%$, and the regular diet group had the lowest rate of $84.02 \%$. Conclusion: The appetite of hospitalized patients is closely related to nutritional status, and therefore, attention should be paid to the appetite status and nutritional status of hospitalized patients. Inpatients with different nutritional support methods should be given individualized appetite and nutritional interventions.

\section{Keywords}

Cross-Sectional Study, Appetite, Nutritional Status, Nutritional Support

\section{Introduction}

Taking a fairly important position, nutritional support treatment runs through the whole process of clinical treatment, and it has been paid more and more attention by medical workers and patients. Appetite is a subjective feeling that gets jointly regulated by the arcuate nucleus of the hypothalamus, the central neurotransmitters of the nucleus of the solitary tract and peripheral hormones [1]. Appetite is closely related to nutrition and is considered to be an intermediate bridge between energy intake and consumption. Loss of appetite can cause weight loss. Hospitalized patients generally have short-term or long-term changes in appetite status due to factors such as disease, surgery, and stress. According to reports, loss of appetite is commonly seen in hospitalized patients, especially cancer patients [2] [3]; appetite assessment is extremely important to ensure patient nutritional support and improve nutritional status. There are few clinical studies on appetite assessment of hospitalized patients and its relationship with nutrition in China. This study selected a tertiary A general hospital in Guangzhou, Guangdong Province to conduct a cross-sectional survey on November 25, 2020 , in order to understand hospitalized patients' appetite and its relations to their nutrition, and provide a basis for the implementation of clinical nutrition support treatment and nursing.

\section{Materials and Methods}

\subsection{Research Subject}

This study is a single-center cross-sectional survey. A tertiary A general hospital in Guangzhou, Guangdong Province was selected as the survey site. A total of 44 wards were surveyed (except emergency department, pediatrics, maternity area, and obstetrics). Inclusion criteria for survey subjects: 1) Patients who have been 
hospitalized for more than 48 hours; 2) Sober patients; 3) Those who have cognitive and comprehension skills and can communicate normally; 4) Patients voluntarily participate in this study. Exclusion criteria: 1) Patients with communication difficulties and unable to answer questions; 2) Patients with lack of basic information or survey data missing; 3) Unable and (or) incapable to cooperate in completing the survey; 4) Patients that do not have more than three of the following index records or results within one week: weight, albumin, hemoglobin, and C-reactive protein.

According to literature review, the sample size should be 5 to 10 times of the number of questionnaire items used in the study, which should be expanded by $20 \%$ to prevent insufficient samples caused by invalid questionnaires. In this study, 17 questionnaire items were used and at least 204 samples should be calculated. The final sample size of this study was 865 cases.

\subsection{Research Tools}

1) General information questionnaire: Including the patient's age, gender, height, weight, diagnosis, length of hospital stays, etc.

2) Appetite assessment scale: The appetite assessment scale uses a $10 \mathrm{~cm}$ long line segment with scales divided into 10 equal parts; " 0 points" means "the worst appetite, no appetite at all", and " 10 points" means "the best appetite", while the rest is between 0 and 10. The patient chooses the value according to his/her own situation. This method is simple, easy, objective and sensitive [4] [5]. Result judgment: scores $<2$ are seen as severe anorexia, scores between 2 and 4 are considered moderate anorexia, between 5 and 7 are considered mild anorexia, and between 8 and 10 are referred to normal appetite.

3) Simple diet self-assessment tool (SDSAT): A simple diet self-assessment tool designed by Chinese scholar Cong Minghua [6] is utilized to assess the daily dietary intake of patients. A domestic multi-center study has verified the effectiveness of this SDSAT. Grading level: 1 point: $<300 \mathrm{kcal}$, mainly clear liquid food, no meat, lack of oil; 2 points: $300-600 \mathrm{kcal}$, half liquid food for three meals, no meat, lack of oil; 3 points: $600-900 \mathrm{kcal}$, one full meal and two semi-liquid meals, basically no meat; 4 points: 900 - $1200 \mathrm{kcal}$, two regular full meals and one semi-liquid meal, less meat and less oil; 5 points: $1200-1500 \mathrm{kcal}$, three normal full meals, staple food, meat, eggs, and sufficient fat.

4) Nutritional Risk Screening 2002 (NRS2002): NRS 2002 is a nutritional risk screening tool developed by the European Society of Parenteral and Enteral Nutrition in 2003 based on 128 randomized controlled studies [7]. NRS2002 includes three parts: nutritional status score, disease severity score and age score; a total score $\geq 3$ points means there is nutritional risk, and $<3$ means no nutritional risk. Both the European Society of Parenteral and Enteral Nutrition and the Chinese Society for Parenteral and Enteral Nutrition recommend NRS2002 as the preferred tool for nutritional risk screening in hospitalized patients [8]. NRS2002 has the advantages of non-invasiveness, time-saving, simplicity, etc., and has 
been proven to have good reliability and validity. The adaptability of hospitalized patients in China to this tool is 95.7\% [9].

5) Nutrition-related indicators: including body weight, body mass index (BMI), grip strength, albumin, hemoglobin, C-reactive protein, etc. The above indicators are based on the patient's test results in the last week of the survey day. BMI = weight $(\mathrm{kg}) /$ height $^{2}\left(\mathrm{~m}^{2}\right)$; when the patient's BMI is less than 18.5 , it is diagnosed as malnutrition. Grip strength: CAMRY brand grip strength meter (model: EH101) is used for grip strength measurement on the inspection day, unit: $\mathrm{Kg} / \mathrm{lb}$ (pound force), suitable for patients $\geq 10$ years old. Let the patient hold the dynamometer with the dominant hand, adjust the grip distance appropriately according to the patient's hand type; palm inward, index dial outward, body upright, arms droop naturally; avoid contact between the dynamometer and the body and the clothing; try to avoid the hand with peripheral vascular infusion; measure 3 times in total, with the interval of each measurement $10 \mathrm{~s}$ or more, and record the average value.

6) Daily intake total calorie compliance rate [10] (hereinafter referred to as daily intake compliance rate): the rate of cases where energy intake $\geq 60 \%$ of the target calories on the day before the patient's investigation; target calories = standard body weight $\times 25 \mathrm{kcal} /(\mathrm{kg} \cdot \mathrm{d})$.

7) Implementation of nutritional intervention: whether to apply for a nutritional consultation, whether to give nutritional support and support methods (oral nutritional supplement, oral feeding, tube feeding, parenteral nutrition), whether to use appetite-improving drugs or methods.

\subsection{Investigation Methods}

The nutrition nursing team of the hospital was responsible for providing survey methods training to the nutrition liaison nurses from different wards. The nutrition liaison nurses completed the hospital-wide cross-sectional survey on November 25,2020 . The nutrition and nursing team members summarized the data and conducted the statistical analysis, and the correctness was cross-checked by two persons. The data that did not meet the standard were excluded, to ensure the quality of the survey results.

\subsection{Statistical Processing}

SPSS 20.0 and EXCEL 2017 were used for statistical analysis of the data. Measurement data or continuous variables were represented by $\bar{x} \pm s$, normal distributions between groups were compared by t-test or analysis of variance, and non-normal distributions were described by rank sum test. Count data or categorical variables were expressed by rate, and statistics were performed by using $x^{2}$ test or Fisher's exact probability method. SpeaHnan correlation analysis and multiple linear regression were used to analyze the correlation between appetite and nutritional indicators in hospitalized patients. $\mathrm{P}<0.05$ was considered statistically significant. 


\section{Results}

A total of 890 survey results were collected, yet 25 cases were entered and reviewed with data missing of more than 3 items, and therefore 865 cases were included in the statistics after the exclusion process.

\subsection{General Information of the Patients}

Among the 865 patients, 288 were in internal medicine departments, 307 were in surgery departments, 40 were in neuro-rehabilitation departments, 58 were in infectious disease department, 117 were in psychiatry department, and 55 were in other departments; 366 were women (42.31\%) and 499 were men (57.69\%); the average age was $(49.02 \pm 19.01)$ years old, $64.73 \%$ were under 18 years old, $80.81 \%$ were 18 - 70 years old, and $12.72 \%$ are $70-88$ years old; height $(1.63 \pm$ $11.47) \mathrm{m}$, weight $(60.23 \pm 14.17) \mathrm{kg}$, BMI $\left(22.48 \pm 4.18 \mathrm{~kg} / \mathrm{m}^{2}\right)$; average hospital stay $(10.67 \pm 11.38)$ days (Table 1$)$.

\subsection{Appetite and Dietary Status}

The average appetite assessment score of the 865 patients was $(6.99 \pm 2.43)$, and $15.84 \%$ had an appetite assessment score of less than 5 ; there were 52 patients who used appetite-improving drugs. Among the 137 patients with an appetite assessment score of less than 5, only 7 patients utilized medications that improve appetite (Table 2). The dietary self-evaluation scores of patients (852 cases) were averagely (4.08 \pm 1.16$)$ points. There were 13 patients who were fasted (Table 3 ).

\subsection{Nutritional Status and Nutritional Support}

Among the 865 patients, the incidence of nutritional risk was $28.67 \%$ (248/865), and the incidence of malnutrition was $13.29 \%$ (115/865). See Table 4 for details.

Table 1. Demographic characteristics of the study sample $(n=865)$.

\begin{tabular}{lcc}
\multicolumn{1}{c}{ Item } & Num. of cases & Ratio \\
\hline Departments & & \\
medicine & 288 & 33.29 \\
surgery & 307 & 35.49 \\
neuro-rehabilitation & 40 & 4.62 \\
infectious disease & 58 & 6.71 \\
psychiatry department & 117 & 13.53 \\
others & 55 & 6.36 \\
Gender & & \\
male & 499 & 42.31 \\
female & 366 & 57.69 \\
Age & & \\
$<18$ yr & 56 & 6.47 \\
$18-70$ yr & 699 & 80.81 \\
$70-88$ yr & 110 & 12.72 \\
\hline
\end{tabular}


Table 2. Appetite of inpatients $(n=865)$.

\begin{tabular}{ccc}
\hline Appetite score & Num. of cases & Percentage (\%) \\
\hline $8-10$ & 413 & 47.75 \\
$5-7$ & 277 & 32.02 \\
$2-4$ & 124 & 14.34 \\
$<2$ & 13 & 1.50 \\
\hline
\end{tabular}

Table 3. Dietary status of the hospitalized patients $(n=852)$.

\begin{tabular}{ccc}
\hline Self-evaluation score & Num. of cases & Ratio \\
\hline 1 & 34 & 3.99 \\
2 & 77 & 9.04 \\
3 & 104 & 12.21 \\
4 & 205 & 24.06 \\
5 & 432 & 50.70 \\
\hline
\end{tabular}

Among patients with nutritional risk, the utilization rate of nutritional support was $60.48 \%(150 / 248) ; 3.24 \%(22 / 248)$ of these patients started tube feeding enteral nutrition, $7.05 \%$ (61/248) started parenteral nutrition, and $10.52 \%(91 / 248)$ implemented oral nutritional supplements. The daily intake compliance rate of the patients was $85.78 \%$ (742/865). 23 of the 865 patients applied for nutrition consultation, of which 11 cases were for physician nutrition consultation, and 12 were for specialized nurse nutrition consultation; the patients who applied for nutrition consultation accounted for $9.27 \%$ (23/248) of the patients with nutrition risk. See Table $4 \&$ Table 5 for details.

\subsection{Relations between the Patients' Appetite and Their Dietary Intake as Well as Nutritional Status}

The appetite assessment scores of patients were strongly correlated with the diet self-evaluation scores and the daily intake compliance rate, and the difference was statistically significant $\left(r_{1}=0.548, r_{2}=0.263, p<0.01\right)$. The differences in body weight, BMI, grip strength, albumin, and hemoglobin concentration of patients with different appetite states were statistically different $(p<0.01$, excluding patients younger than 10 years old, $\mathrm{n}=827$ ), see Table 6 for details. Taking appetite as an independent variable and weight, BMI, grip strength, albumin, and hemoglobin as dependent variables for multiple linear regression analysis, the results showed that appetite was an influencing factor of weight change ( $\beta=$ $-0.079, p=0.023)$.

\subsection{Relations between Appetite and Daily Intake Compliance Rate of Patients with Different Nutritional Support Methods}

Patients were divided into 5 groups according to different nutritional support methods, including parenteral nutrition group, tube feeding nutrition group, diet 
Table 4. Nutritional status and nutritional support intervention status of hospitalized patients $(\mathrm{n}=865)$.

\begin{tabular}{ccc}
\hline Item & Num. of cases & Total incidence (\%) \\
\hline Nutritional risk & 248 & 28.67 \\
Malnutrition & 115 & 13.29 \\
Daily intake compliance & 742 & 85.78 \\
Nutritional support & 150 & 17.34 \\
Nutrition consultation & 23 & 2.66 \\
\hline
\end{tabular}

Table 5. Nutritional support interventions for patients at nutritional risk $(\mathrm{n}=248)$.

\begin{tabular}{lcc}
\hline \multicolumn{1}{c}{ Item } & Num. of cases & Utilization rate (\%) \\
\hline Tube feeding nutrition & 16 & 6.45 \\
Parenteral nutrition & 40 & 16.13 \\
Diet + ONS & 71 & 28.63 \\
Tube feeding + ONS & 2 & 0.81 \\
Parenteral nutrition + ONS & 17 & 6.85 \\
Tube feeding + parenteral nutrition & 4 & 1.61 \\
Individualized diet & 14 & 5.65 \\
Regular diet & 84 & 33.87 \\
\hline
\end{tabular}

Table 6. Comparison of body weight, BMI, grip strength, albumin and hemoglobin concentration of patients with different appetites $(\bar{x} \pm s, \mathrm{n}=827)$.

\begin{tabular}{ccccccc}
\hline $\begin{array}{c}\text { Appetite } \\
\text { assessment score }\end{array}$ & Num. of cases & $\begin{array}{c}\text { Weight } \\
(\mathbf{k g})\end{array}$ & $\begin{array}{c}\text { BMI } \\
\left(\mathbf{k g} / \mathrm{m}^{2}\right)\end{array}$ & $\begin{array}{c}\text { Grip strength } \\
(\mathrm{kg})\end{array}$ & $\begin{array}{c}\text { Albumin } \\
(\mathrm{g} / \mathrm{L})\end{array}$ & $\begin{array}{c}\text { Hemoglobin } \\
(\mathrm{g} / \mathrm{L})\end{array}$ \\
\hline$<2$ & 13 & $56.08 \pm 12.39$ & $20.23 \pm 3.68$ & $21.15 \pm 6.88$ & $37.23 \pm 5.49$ & $113.69 \pm 18.87$ \\
$2-4$ & 124 & $58.05 \pm 12.29$ & $21.84 \pm 4.72$ & $21.26 \pm 7.78$ & $37.66 \pm 5.54$ & $114.94 \pm 21.58$ \\
$5-7$ & 277 & $60.13 \pm 13.19$ & $22.25 \pm 3.96$ & $25.18 \pm 12.54$ & $39.92 \pm 6.82$ & $117.97 \pm 26.59$ \\
$8-10$ & 413 & $62.70 \pm 13.24$ & $23.22 \pm 3.90$ & $30.29 \pm 15.25$ & $40.35 \pm 5.50$ & $124.75 \pm 22.46$ \\
$F$ value & & 3.043 & 4.241 & 6.431 & 4.741 & 4.395 \\
$P$ value & & $<0.001$ & $<0.001$ & $<0.001$ & $<0.001$ & $<0.001$ \\
\hline
\end{tabular}

+ ONS group, individualized diet group and routine diet group. The patients receiving parenteral nutrition were classified into a group with 61 patients; the patients receiving tube feeding nutrition were classified into a group (excluding patients receiving parenteral nutrition at the same time) with 18 patients; patients receiving diet + ONS, individualized diet guidance as well as regular diet were classified into their respective groups. The difference in appetite assessment score and daily intake compliance rate of patients with different nutritional support methods was statistically significant $(p<0.05)$. Among the 3 groups of patients who can eat orally, the individualized diet guidance group had signifi- 
cantly higher appetites than the non-intervention regular diet group and the diet + ONS group, and the difference was statistically significant $(p<0.05)$. The highest compliance rate of daily intake in the individualized diet guidance group was $100 \%$, followed by the parenteral nutrition group at $96.72 \%$, and the lowest was in the regular diet group at $84.02 \%$ (Table 7).

\section{Discussion}

\subsection{The Appetite and Nutritional Status of Hospitalized Patients Are Poor, and the Implementation of Nutritional Support Is Still Not Ideal}

Nutritional risks or malnutrition problems are common among hospitalized patients. It is reported in the literature that $20 \%-60 \%$ of patients have nutritional risks when they are admitted to the hospital, and $30 \%-80 \%$ of patients have significant body weight loss during hospitalization [11]. Malnutrition leads to longer hospital stays, increased complications and medical costs, reduced quality of life, and increased mortality [12] [13]. In this cross-sectional survey, 28.67\% of the patients had nutritional risk, and $14.22 \%$ of the patients had substandard daily nutrition intake. It can be seen that although the incidence of nutritional risk in hospital inpatients under investigation is lower than that reported in the literature, it is still relatively high and there are still related problems. A lot of evidence shows [14] [15] that nutritional intervention can improve the clinical outcome of patients and improve the overall quality of life of patients. However, in this study, patients with nutritional support (including tube feeding, ONS and parenteral nutrition) accounted for only $60.48 \%$ of the patients at nutritional risk, while patients who applied for nutritional consultation accounted for only $9.27 \%$ of the patients at nutritional risk. In addition, the average appetite assessment score of 865 patients was $(6.99 \pm 2.43)$, which was at the level of mild anorexia; patients with mild anorexia (appetite score $<8$ ) accounted for $50.06 \%$ (414/827), but only 7 patients were treated with medications to improve appetite. The results of this study show that the current nutritional support and appetite

Table 7. Analysis of appetite and daily intake compliance rate of patients with different nutritional support methods $(\mathrm{n}=865)$.

\begin{tabular}{ccccc}
\hline Group & Num. of cases & $\begin{array}{c}\text { Daily intake compliance } \\
\text { rate (\%) }\end{array}$ & \multicolumn{1}{c}{ Appetite assessment score (points, $\bar{x} \pm s)$} \\
\cline { 4 - 5 } & & $96.72(59 / 61)$ & Average points & $p$ value between groups \\
\hline Parenteral nutrition & 61 & $88.89(16 / 18)$ & $4.90 \pm 2.99$ & 0.003 (Group 3 \& 4) \\
Tube feeding nutrition & 18 & $90.14(64 / 71)$ & $6.49 \pm 2.20$ & 0.017 (Group 4 \& 5) \\
Diet + ONS & 71 & $100.00(14 / 14)$ & $8.00 \pm 2.74 \pm 1.70$ & 0.030 (Group 3 \& 5) \\
Individualized diet & 14 & $84.02(589 / 701)$ & $7.19 \pm 2.30$ & $15.770^{2)}$ \\
Regular diet & 701 & $-1)$ & & 0.000 \\
\hline
\end{tabular}

${ }^{\star}$ Note: 1) refers to Fisher's exact probability test, 2) refers to $F$ value. 
intervention status of hospitalized patients are not ideal. Although most medical staff have recognized the importance of nutritional support treatment, they have not been able to integrate patient nutritional support into clinical practice in actual work [16]. Therefore, it is necessary to further strengthen the awareness of clinical medical staff to pay attention to the nutritional status of patients and timely implement nutritional support, and to pay more attention to the appetite status of patients, and provide diversified nutritional support methods to meet the nutritional needs of patients.

\subsection{Appetite of Hospitalized Patients Is Closely Related to Their Nutritional Status}

The results of this study show that appetite is correlated with dietary intake score and daily intake compliance rate $(p<0.01)$; the higher the appetite assessment score, the higher the values of weight, BMI, albumin, grip strength, and hemoglobin concentration of patients, and the difference is statistically significant $(p<$ 0.01 ). This indicates that the appetite of hospitalized patients is closely related to the body weight, BMI, grip strength, albumin, and hemoglobin concentration; and appetite is a predictive factor for weight changes $(\beta=-0.079, \mathrm{p}=0.023)$, which is consistent with the research results of Landi et al. and Li et al. [16] [17], providing a certain theoretical basis for the application and promotion of appetite assessment in the nutritional care of inpatients. Appetite directly affects food intake, so appetite is closely related to insufficient nutritional intake and weight loss, and is one of the important factors predicting nutritional risk [18]. Therefore, appetite assessment and intervention are very important to improve the nutritional status of hospitalized patients. Appetite assessment must be done early; timely and effective interventions based on the appetite assessment results can help reduce the occurrence of malnutrition. Scientific appetite evaluation methods and technologies can not only provide nutrition workers with tools to objectively evaluate appetite, but also provide a basis for further understanding and predicting malnutrition [19]. However, currently medical staff do not pay enough attention to appetite evaluation and intervention, and they also lack the scientific assessment tools for the appetite assessment and monitoring of patients. The survey also found that most departments still lack standardization and proficiency in the use of appetite assessment scales. The standardization of the appetite assessment tools and their use methods need to be further regulated.

\subsection{Inpatients with Different Nutritional Support Methods Should Be Given Individualized Appetite and Nutritional Interventions}

The results of this study show that the appetite scores of patients with different nutritional support methods are different, and the difference is statistically significant $(p<0.05)$. The individualized diet group had the highest appetite score, reaching $(8.57 \pm 1.70)$ points, which was significantly higher than that of the regular diet group and the diet + ONS group. The individualized diet group 
achieved the highest intake compliance rate at the same time. It can be seen that individualized dietary education can improve the appetite of patients. Pictures and language related to food or eating during the education process may stimulate the patient's conditioned reflex to eat, stimulate the secretion of saliva and gastric juice, and then produce appetite [20]. In addition, nutritional cognition directly affects people's food choices; inappropriate food choices, artificially narrowing the range of food choices, or patients having post-eating uncomfortable reactions, will all affect their nutritional intake [21]. Inpatients often change their eating habits due to psychological shocks or restrictions in the inpatient environment. Therefore, it is extremely important to correct the nutritional cognition of patients through dietary education, which can indirectly increase the patients' nutrition intake and nutritional level. The low appetite scores of patients with parenteral nutrition and tube feeding may be related to reduced appetite caused by illness, fasting, inadaptability of parenteral nutrition, gastrointestinal intolerance symptoms, discomfort of indwelling nutrition infusion tube and other reasons. This suggests that attention should be paid to the appetite status of these patients in clinical treatment [22], and whether the nutritional status can be improved by improving the appetite status of these patients remains to be further studied.

In addition, the daily total calorie intake compliance rate of the patients in each group is ranked from high to low as follows: individualized diet group, parenteral nutrition group, diet + ONS group, tube feeding nutrition group, and lastly, regular diet group. The difference is statistically significant $(p<0.05)$. This indicates that individualized dietary guidance has a better nutritional intervention effect, and parenteral nutrition can also achieve a higher rate of nutritional intake. Therefore, based on the goal of meeting the patient's daily total calorie intake, it is necessary to strengthen the evaluation of patients; for patients whose total intake is not up to standard, further nutritional intervention measures should be given according to the condition; to improve dietary compliance, individualized dietary education should be enhanced for patients who can eat orally [23]. Studies have shown that nutrition consultation allows patients to receive scientific and reasonable dietary guidance, which is beneficial to improve the quality of life of patients. This effect may exceed the impact on the nutritional status of patients [24], so it can be seen that the role of nutrition consultation is very crucial. Although some studies believe that parenteral nutrition will increase the incidence of nosocomial infections, the pros and cons should be weighed in light of the actual situation of the patient [25]. The implementation of tube feeding may be limited by the patient's gastrointestinal function. The implementation generally has fewer constraints, and the full dose can be used unless the patient has poor liver function or financial difficulties. Therefore, when the patient's gastrointestinal function cannot meet the full calorie supply, parenteral nutrition should be considered to ensure the total nutritional intake and meet the body's needs, which will help speed up the recovery of the disease [26]. In addition, this survey shows that nutritional treatment planning have 
been made as part of the overall treatment plan, but medical workers often fail to implement effective nutritional interventions; the nutritional treatments lack individualized programs, and nutritional compliance situation is not satisfactory; the nutritional education for inpatients is not good, and the appetite evaluation and monitoring of patients are not paid enough attention. It is necessary to study a reasonable nutrition and appetite treatment process so as to standardize clinical work.

This study also has certain limitations. As a short-term cross-sectional study, this survey failed to continuously observe the patient's nutrition and prognosis, so the survey results cannot accurately reflect appetite and nutritional intervention during the entire course of the disease. Moreover, the survey content is relatively narrow and one-sided, and it is difficult to conduct a systematic and comprehensive analysis on certain phenomenon or the cause of the results.

\section{Conclusion}

The appetite of hospitalized patients is closely related to nutritional status, and therefore, attention should be paid to the appetite status and nutritional status of hospitalized patients. Inpatients with different nutritional support methods should be given individualized appetite and nutritional interventions.

\section{Fund Project}

Guangdong Provincial Medical Research Fund (A2018398), Guangdong Provincial Nursing Society Fund (gdhlxueh2019zx077).

\section{Conflicts of Interest}

The authors declare no conflicts of interest regarding the publication of this paper.

\section{References}

[1] Davis, M.P., Dreicer, R., Walsh, D., et al. (2004) Appetite and Cancer-Associated Anorexia: A Review. Journal of Clinical Oncology, 22, 1510-1517. https://doi.org/10.1200/JCO.2004.03.103

[2] Wilkes, G.M. (1995) Cancer and HIV Clinical Nutrition Pocket Guide. Wilkes Jones and Bartlett Publishers, Boston.

[3] Bennani-Baiti, N. and Walsh, D. (2009) What Is Cancer Anorexia-Cachexia Syndrome? A Historical Perspective. Journal of the Royal College of Physicians of Edinburgh, 39, 257-262.

[4] Wen, S. and Liu, H.Q. (2020) Progress on Pathogenesis, Clinical Diagnosis and Treatment of Cancer-Related Anorexia. Chinese Journal of Clinical Oncology, 47, 1013-1018.

[5] Ma, H.X. and Li, S.Y. (2018) The Mechanism, Diagnosis and Treatment of Cancer-Related Anorexia. Electronic Journal of Metabolism and Nutrition of Cancer, $\mathbf{5}$ 117-121.

[6] Cong, M.H. and Shi, H.P. (2020) The Invention of a Simple Diet Self-Assessment 
Tool for Cancer Patients. Electronic Journal of Metabolism and Nutrition of Cancer, 5, 11-13.

[7] Kondrup, J., Allison, S.P., Elia, M., et al. (2003) ESPENF Guidelines for Nutrition Screening 2002. Clinical Nutrition, 22, 415-421. https://doi.org/10.1016/S0261-5614(03)00098-0

[8] Chinese Medical Association (2007) Clinical Diagnosis and Treatment GuidelinesParenteral Enteral Nutrition. People's Medical Publishing House, Beijing, 18-20.

[9] Xu, J.Y., Yang, J., Kang, W.M., et al. (2018) Clinical Expert Consensus on Nutritional Risk and Nutritional Risk Screening Tool (Nutritional Risk Screening 2002). Chinese Journal of Clinical Nutrition, 26, 131-135.

[10] Xing, J., Zhang, Z.H., Ke, L., et al. (2019) A Cross-Sectional Study on Nutritional Status of ICU Patients in the Mainland China. Medical Journal of Chinese People's Liberation Army, 44, 388-393.

[11] Löser, C. (2010) Malnutrition in Hospital: The Clinical and Economic Implications. Deutsches Ärzteblatt International, 107, 911-917.

[12] Leiva, B.E., Badia, T.M., Virgili, C.N., et al. (2017) Hospital Malnutrition Screening at Admission: Malnutrition Increases Mortality and Length of Stay. Nutricion Hospitalaria, 34, 907-913.

[13] Curtis, L.J., Bernier, P., Jeejeebhoy, K., et al. (2017) Costs of Hospital Malnutrition. Clinical Nutrition, 36, 1391-1396. https://doi.org/10.1016/j.clnu.2016.09.009

[14] Baldwin, C. (2015) The Effectiveness of Nutritional Interventions in Malnutrition and Cachexia. Proceedings of the Nutrition Society, 74, 397-404. https://doi.org/10.1017/S0029665115002311

[15] Lis, C.G., Gupta, D., Lammersfeld, C.A., et al. (2012) Role of Nutritional Status in Predicting Quality of Life Outcomes in Cancer a Systematic Review of the Epidemiological Literature. Nutrition Journal, 11, 27. https://doi.org/10.1186/1475-2891-11-27

[16] Li, Z.J., Yao, Y.X., Li, H.L., et al. (2016) An Overview Analysis of Cancer-Related Malnutrition in Hospitalized Patients: A Multicenter Cross-Sectional Study. Chinese Journal for Clinicians, 44, 19-23.

[17] Landi, F., Liperoti, R., Latanzio, F., et al. (2012) Effects of Anorexia on Mortality among Older Adults Receiving Home Care: An Observation Study. The Journal of Nutrition, Health and Aging, 16, 79-83. https://doi.org/10.1007/s12603-011-0064-y

[18] Mudge, A.M., Ross, L.J., Young, A.M., et al. (2011) Helping Understand Nutritional Gaps in the Elderly (HUNGER): A Prospective Study of Patient Factors Associated with Inadequate Nutritional Intake in Order Medical Inpatients. Clinical Nutrition, 30, 320-325. https://doi.org/10.1016/j.clnu.2010.12.007

[19] Du, H.Z. (2017) Evaluation of Nutritional Status and Appetite in Patients with Malignant Tumor. Hebei Medical University, Shijiazhuang, 1-58.

[20] Li, D.Y., Sun, Y.B., Qi, Y.H., et al. (2018) Effect of Appetite Condition Reflex Stimulation on Tolerance of Early Enteral Nutrition after Colorectal-Carcinoma Surgery. Electronic Jourmal of Metabolism and Nutrition of Cancer, 5, 366-371.

[21] Zhao, W.Z., Ji, L.L., Yu, K.Y., et al. (2020) Compilation and Reliability and Validity Test of Nutrition Belief-Appetite-Function Scale for Cancer Patients. Electronic Jourmal of Metabolism and Nutrition of Cancer, 7, 421-427.

[22] Li, Z.N., Li, X.L., Chen, W., et al. (2020) Expert Consensus on Appetite Assessment and Regulation in Cancer Patients. Electronic Journal of Metabolism and Nutrition of Cancer, 7, 169-177. 
[23] Shi, H.P., Yang, J. and Zhang, Y. (2017) Nutrition Education for Cancer Patients. Electronic Jourmal of Metabolism and Nutrition of Cancer, 4, 1-6.

[24] Poulsen, G.M., Pedersen, L.L., Osterlind, K., et al. (2014) Randomized Trial of the Effects of Individual Nutritional Counseling in Cancer Patients. Clinical Nutrition, 33, 749-753. https://doi.org/10.1016/j.clnu.2013.10.019

[25] Pradelli, L., Graf, S., Pichard, C., et al. (2018) Supplemental Parenteral Nutrition in Intensive Care Patients: A Cost Saving Strategy. Clinical Nutrition, 37, 573-579. https://doi.org/10.1016/j.clnu.2013.10.019

[26] Wang, L., Yang, L.Q., Li, S.Y., et al. (2020) Construction of Malnutrition Management System in Hospital: An Introduction to Hunger-Free Hospital Programme. Electronic Journal of Metabolism and Nutrition of Cancer, 7, 381-386. 